\title{
La liberté religieuse en droit français actuel. Un point de vue chrétien
}

Alain Sériaux

\section{OpenEdition}

1 Journals

Édition électronique

URL : https://journals.openedition.org/rdr/1258

DOI : $10.4000 /$ rdr.1258

ISSN : 2534-7462

Éditeur

Presses universitaires de Strasbourg

\section{Édition imprimée}

Date de publication : 16 novembre 2020

Pagination : 157-172

ISBN : 979-10-344-0073-7

ISSN : 2493-8637

Référence électronique

Alain Sériaux, "La liberté religieuse en droit français actuel. Un point de vue chrétien », Revue du droit des religions [En ligne], 10 | 2020, mis en ligne le 16 novembre 2020, consulté le 02 mai 2022. URL: http://journals.openedition.org/rdr/1258; DOI : https://doi.org/10.4000/rdr.1258

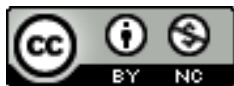

La revue du droit des religions est mise à disposition selon les termes de la Creative Commons Attribution - Pas d'Utilisation Commerciale 4.0 International - CC BY-NC 4.0. 


\section{LALIBERTÉRELIGIEUSEEN DROITFRANÇAIS ACTUEL.UN POINT DEVUECCHRÉTIEN}

\section{Alain SÉRIAUX}

Laboratoire de droit privé et de sciences criminelles (LDPSC), Aix-Marseille Université

\section{Résumé}

Aspect principal de la liberté de conscience, la liberté religieuse ne se limite pas au pur domaine du for interne. Il est aussi de sa nature de s'extérioriser par des actes de culte, des signes d'appartenance et autres symboles physiquement identifiables. Or, dans la société française contemporaine, sous l'influence de divers facteurs, le statut juridique de la religion au for externe est devenu problématique. Jamais le droit de critique à son encontre n'a été aussi garanti et protégé par le droit; jamais n'ont autant prospéré le principe de laicité et le confinement de la foi au seul domaine de l'intime qu'il emporte. Ce double phénomène affecte toutes les religions en France, mais il ne les affecte pas au même degré, ni de la même manière. Voici un point de vue chrétien.

\section{Abstract}

Religious freedom, the principal aspect of freedom of conscience, is not limited to the pure domain of the internal forum. It is also of its nature to be exteriorized by worship, signs of belonging and other identifiable material symbols. However, in contemporary French society, under the influence of various factors, the legal status of religion in its external forum has become problematic. Never before has the right to criticize it been so guaranteed and protected by law; never before has the principle of laïcité and the confinement of faith to the intimate realm flourished so much. This dual phenomenon affects all religions in France, but it does not affect them to the same degree or in the same way. Here is a Christian point of view. 
A ujourd'hui, en France, il ne se passe presque pas de semaine sans que des 1 questions relatives à la liberté religieuse ne soient commentées ou débattues dans les médias. C'est, comme on le sait, l'islam qui occupe le devant de la scène. Le christianisme, lui, a fait son temps, qui nous renvoie à divers épisodes célèbres de notre histoire, notamment la loi du 9 décembre 1905 sur la séparation des Églises et de l'État. L'intérêt contemporain pour l'islam et l'oubli corrélatif de la religion chrétienne tiennent à divers facteurs. Sociologiquement, la religion fondée par Mahomet occupe désormais chez nous une place de plus en plus conséquente. S'il n'existe pas, sur la religion des Français, de statistiques officielles - elles sont interdites par l'article 6-I de la loi 6 janvier 1978 relative à l'informatique, aux fichiers et aux libertés -, des chiffres qui remontent au début des années 2010 donnaient, au sein de la population française, $7,50 \%$ de musulmans et $66 \%$ de chrétiens (dont $54 \%$ de catholiques). Or, déjà à cette époque, parmi les personnes interrogées, $41 \%$ de personnes "d'origine musulmane» se disaient "croyantes et pratiquantes» contre $16 \%$ seulement chez les catholiques ${ }^{1}$. De plus, chez ces derniers, seuls $4,5 \%$ se rendaient en réalité régulièrement à la messe dominicale, alors que, du côté musulman, $25 \%$ des interrogés disaient aller «généralement à la mosquée le vendredi ${ }^{2}$. Tout porte donc à croire qu'en 2019, même si la pratique religieuse a régressé de part et d'autre, l'islam est devenu le premier culte pratiqué en France. Par ailleurs, sur l'échiquier politique cette fois, la confession musulmane est devenue, même si elle ne s'avoue pas telle, un véritable enjeu électoral. Elle occupe aussi une place considérable parmi les préoccupations de police intérieure, avec le développement de l'islamisme radical. Face à l'islam, gouvernants et médias avancent avec circonspection, tandis qu'à l'égard du christianisme, ils ont à tort ou à raison le sentiment de se trouver sur un terrain bien connu qui les dispense de toute attention particulière.

Mais là où les deux religions se rejoignent et sont forcément placées sur un pied d'égalité, c'est dans leur traitement juridique. Comme n'importe quelle autre profession de foi religieuse, elles sont soumises au droit français des religions, qui se rattache à la tradition des démocraties modernes prônant un principe de liberté religieuse. À la suite de l'article 18 de la Déclaration universelle des droits de l'homme de 1948, la Convention européenne des droits de l'homme et des libertés fondamentales de 1950 proclame en son

1. A. Pouchard et S. Laurent, "Quel est le poids de l'Islam en France?», Le Monde, 21 janvier 2015.

2. L. MARChAND, «Plus de la moitié des Français ne se réclament d'aucune religion», Le Monde, 7 mai 2015. 
article 9: "Toute personne a droit à la liberté de pensée, de conscience et de religion; ce droit implique la liberté de changer de religion ou de conviction, ainsi que la liberté de manifester sa religion ou sa conviction individuellement ou collectivement, en public ou en privé, par le culte, l'enseignement, les pratiques et l'accomplissement des rites. » Ratifiée par la France depuis 1974, cette Convention s'applique directement dans notre ordre juridique interne. La Cour européenne des droits de l'homme est particulièrement chargée de veiller à son respect par les autorités nationales. La France a de toute façon son propre dispositif national qui abonde largement dans le même sens. Aux termes de l'article $1^{\text {er }}$ de la loi du 9 décembre 1905, «La République assure la liberté de conscience. Elle garantit le libre exercice des cultes sous les seules restrictions édictées ci-après dans l'intérêt de l'ordre public ». Dans un registre comparable, l'article $1^{\text {er }}$, alinéa $1^{\text {er }}$, de la Constitution du 4 octobre 1958 énonce pour sa part: "La France est une République indivisible, laïque, démocratique et sociale. Elle assure l'égalité devant la loi de tous les citoyens sans distinction d'origine, de race ou de religion. Elle respecte toutes les croyances. » En somme, comme beaucoup d'autres nations où, en général, il fait bon vivre, la France s'engage à protéger la liberté de croire et de pratiquer sa foi, peu importe laquelle, dès lors que cette pratique ne porte pas atteinte à l'ordre public et aux bonnes mœurs. Elle veille simplement, en sus, à ce que chacun, sous prétexte de zèle religieux, n'impose pas ses croyances aux autres.

Voilà qui, à plus d'un titre, devrait faciliter, chez les disciples du Christ, la réalisation de leur vocation religieuse dans ce qu'elle a de plus authentique. La religion chrétienne se caractérise avant tout par son intériorité. Loin d'être la simple adhésion à une série de dogmes, elle trouve sa plénitude dans une rencontre personnelle avec le Christ, identifié comme Fils de Dieu. Tant que cette rencontre n'a pas lieu, la foi au Christ «mort et ressuscité» demeure encore largement informelle, aurait-on été éduqué dans cette croyance dès sa plus tendre enfance. Une telle intériorisation, qui se renforce avec une vie de prière et de fréquentation des sacrements, ne dérange personne: elle regarde exclusivement le croyant. La foi chrétienne a aussi une dimension sociale. Elle suppose la mise en ouvre, par le croyant du message de paix, de justice et d'amour, de don de soi et de pardon, que contiennent les Évangiles. N'est-ce point là une contribution de premier ordre à l'édification d'une vie sociale harmonieuse - le rêve secret de tout gouvernant soucieux du bien commun? Le chrétien qui vit sa foi au quotidien est - ou devrait être - dans la famille, dans la vie professionnelle, dans la vie sociale et politique, un ferment inépuisable d'enrichissement spirituel et de joie pour ceux qui l'entourent. Que, comme tout croyant, le chrétien ambitionne de 
transmettre sa foi à d'autres, voilà qui n'a rien de choquant dès lors qu'il le fait à travers le témoignage d'une vie cohérente, respectueuse de la liberté intérieure de ses interlocuteurs. Ce sont là les bases indispensables d'un pluralisme social bien compris.

Comme toute religion, celle du Christ conduit en définitive à l'adoption d'un certain style de vie, variable selon la vocation personnelle de chacun. Or, c'est précisément cet aspect totalisant (et non totalitaire) de la foi chrétienne qui suscite l'incompréhension, voire la suspicion, dans une société comme la nôtre. Pétries d'athéisme ou d'agnosticisme, les mentalités contemporaines ont du mal à concevoir combien, pour le croyant, l'unité de toute sa vie sous le regard de Dieu est déterminante. Un tel malentendu est d'abord, nous semble-t-il, à l'origine de la difficulté qu'éprouvent actuellement les magistrats à déterminer correctement les limites du droit de critique à l'égard des convictions d'autrui (1). Mais il y a plus. Dans nombre de domaines, cette unité de vie est purement et simplement niée par le droit français qui, au nom d'une conception très dogmatique du principe de laïcité, cherche à refouler les convictions religieuses dans la sphère de la vie privée (2).

\section{LIBERTÉ RELIGIEUSE ET LIBERTÉ D’EXPRESSION}

Dans les démocraties modernes, la liberté d'expression constitue, au même titre que la liberté religieuse, une liberté fondamentale. "Toute personne a droit à la liberté d'expression. Ce droit comprend la liberté d'opinion et la liberté de recevoir ou de communiquer des informations ou des idées sans qu'il puisse y avoir ingérence d'autorités publiques et sans considération de frontière» dispose l'article 10 de la Convention européenne des droits de l'homme. En France, depuis 1789, cette liberté est explicitement conçue comme l'« un des droits les plus précieux de l'homme» (Déclaration des droits de l'homme et du citoyen, art. 11), tandis que la Cour européenne des droits de l'homme voit en elle un «fondement essentiel d'une société démocratique ${ }^{3} »$. Mais d'un autre côté, la même Cour, depuis l'arrêt Kokkinakis c. Grèce du 25 mai 1993, considère la liberté de religion comme "une des assises d'une "société démocratique" au sens de la Convention, [figurant] parmi les éléments les plus essentiels de l'identité des croyants et de leur conception de la vie, mais [qui] est aussi un bien précieux pour les athées, les agnostiques, les sceptiques ou les indifférents. Il y va du pluralisme - chèrement conquis au

3. CEDH, 7 déc. 1976, n 5493/72, Handyside c. Royaume-Uni, § 49. 
cours des siècles - consubstantiel à pareille société ${ }^{4}$ ». Puisqu'il s'agit de deux libertés fondamentales de niveau sensiblement égal, la liberté d'expression des uns devrait pouvoir être contenue dans des limites de nature à respecter la liberté des croyances des autres. Telle est bien la leçon qui s'évince de la jurisprudence de la Cour européenne des droits de l'homme. Dans son arrêt Otto-Preminger-Institut du 20 septembre 19945, elle explique que

«ceux qui choisissent d'exercer la liberté de manifester leur religion, qu'ils appartiennent à une majorité ou à une minorité religieuse, ne peuvent raisonnablement s'attendre à le faire à l'abri de toute critique. Ils doivent tolérer et accepter le rejet par autrui de leurs croyances religieuses et même la propagation par autrui de doctrines hostiles à leur foi. Toutefois, la manière dont les croyances et doctrines religieuses font l'objet d'une opposition ou d'une dénégation est une question qui peut engager la responsabilité de l'État, notamment celle d'assurer à ceux qui professent ces croyances et doctrines la paisible jouissance du droit garanti par l'article 9. En effet, dans des cas extrêmes le recours à des méthodes particulières d'opposition à des croyances religieuses ou de dénégation de celles-ci peut aboutir à dissuader ceux qui les ont d'exercer leur liberté de les avoir et de les exprimer» (§ 46).

Elle en conclut notamment que les États ont «une obligation d'éviter autant que faire se peut des expressions qui sont gratuitement offensantes pour autrui et constituent donc une atteinte à ses droits et qui, dès lors, ne contribuent à aucune forme de débat public capable de favoriser le progrès dans les affaires du genre humain» (§ 49). En somme, dans un débat sur les idées religieuses des uns et des autres, chacun devrait chercher à convaincre l'autre de son erreur, non à vaincre ou, pire encore, à mépriser ou à rabaisser.

Dans un rapport rendu le 23 octobre $2008^{6}$, la Commission européenne pour la démocratie par le droit (Commission de Venise) identifie trois types principaux de manifestations de la liberté d'expression en lien avec la religion condamnés dans les législations européennes: le blasphème; l'injure à caractère religieux; l'incitation à la haine religieuse. Du point de vue du croyant, le blasphème est sans nul doute le comportement le plus gravement répréhensible. Ne constitue-t-il pas, selon la définition retenue par la même

4. CEDH, 25 mai 1993, nº 14307/88, Kokkinakis c. Grèce, § 31.

5. CEDH, 20 sept. 1994, n 13470/87, Otto-Preminger-Institute c. Autriche.

6. Rapport sur les relations entre liberté d'expression et liberté de religion: réglementation et répression du blasphème, de l'injure à caractère religieux et de l'incitation à la haine religieuse, adopté par la Commission de Venise lors de sa $76^{\mathrm{e}}$ session plénière (Venise, 17-18 oct. 2008), étude $\mathrm{n}^{\circ} 406 / 2006$. 
Commission (§ 24) «l'insulte, le mépris ou le manque de respect envers un dieu et, par extension, envers tout ce qui est considéré comme sacré » par telle ou telle religion? Il reste que le blasphème ainsi compris a un statut problématique dans un État qui, comme la France, se veut aconfessionnel ou laïque. Comment un tel État pourrait-il condamner le blasphème, sans affirmer officiellement son acceptation de l'existence d'un Dieu ${ }^{7}$ ? De fait, le délit de blasphème n'est qu'exceptionnellement réprimé en Europe ${ }^{8}$. En France, il a longtemps subsisté, sans jamais être appliqué, dans le Code pénal local d'Alsace-Moselle . Mais l'article 172 de la loi no 2017-86 du 27 janvier 2017 l'a officiellement abrogé. En revanche, l'injure à caractère religieux et l'incitation à la haine religieuse ne sont pas inconnus du droit français. C'est bien dans cette perspective que s'inscrivent les dispositions de la loi du 29 juillet 1881 sur la liberté de la presse lorsqu'elles répriment, respectivement, la diffamation (art. 32) ou l'injure (art. 33) «envers une personne ou un groupe de personnes à raison de leur origine ou de leur appartenance ou de leur nonappartenance à une ethnie, une nation, une race ou une religion déterminée». L'article 24 , alinéa 5 , de la même loi réprime pour sa part la provocation «à la discrimination, à la haine ou à la violence à l'égard d'une personne ou d'un groupe de personnes à raison de [...] leur appartenance [...] à une religion déterminée». De son côté, la jurisprudence civile reconnaît sans difficulté à une association dont l'objet social est de promouvoir et de protéger certaines valeurs religieuses "un intérêt légitime à agir contre une publication qui, selon elle, porte atteinte aux sentiments religieux de ses membres ${ }^{10}$ ». Un tel dispositif n'autorise-t-il pas à penser qu'en droit français, conformément à ce que souhaite la Cour européenne des droits de l'homme, les croyances de chacun, quelle que soit la religion qu'il professe, se trouvent soustraites aux excès de la liberté d'expression? Il faut pourtant y voir de plus près.

7. L'argument n'est peut-être pas imparable, puisqu'aussi bien le préambule de la Déclaration des droits de l'homme de 1789 se place explicitement «sous les auspices de l'Être suprême». Personne, que l'on sache, n'a encore demandé l'abrogation de cette incise.

8. Sur l'ensemble de ces questions, V. C. LAGeot, F. Marchadier (dir.), Le blasphème dans une société démocratique, Paris, Dalloz, 2016.

9. Art. 166: «Celui qui aura causé un scandale en blasphémant publiquement contre Dieu par des propos outrageants, ou aura publiquement outragé un des cultes chrétiens ou une communauté religieuse établie sur le territoire de la Confédération et reconnue comme corporation, ou les institutions ou cérémonies de ces cultes, ou qui, dans une église ou un autre lieu consacré à des assemblées religieuses, aura commis des actes injurieux et scandaleux, sera puni d'un emprisonnement de trois ans au plus.»

10. Cass. $1^{\text {re }}$ civ., 14 nov. 2000 : Bull. civ. I, no 289. À propos d'un livre intitulé INRI, sur la couverture duquel figurait l'image d'une femme nue crucifiée, surmontée de l'inscription «INRI» : parodie pour le moins désobligeante de la crucifixion de Jésus-Christ, telle que les Évangiles la racontent. 
C'est sur le terrain des procédures de référé destinées à empêcher une atteinte imminente aux croyances chrétiennes que nos magistrats se sont longtemps efforcés de trouver un équilibre entre liberté d'expression et liberté religieuse. Ils distinguaient à cet effet selon que les croyants pouvaient ou non se soustraire aux images ou aux propos qui heurtaient leurs convictions personnelles. Nul n'est obligé de lire un article de presse ou un livre, ni de voir un film ou un spectacle. Qu'importent dès lors au croyant les idées professées ou représentées par l'auteur, alors même qu'elles sont objectivement offensantes pour sa foi. Mais qu'en revanche images ou propos soient exposés à la vue de tous, de telle sorte qu'il soit pratiquement impossible d'y échapper, et la protection de la liberté religieuse justifie toute mesure de retrait de l'espace public dès lors qu'elle s'avère proportionnée au but poursuivi ${ }^{11}$. Dans un contexte différent (celui du Tyrol autrichien), la Cour européenne des droits de l'homme, dans son arrêt Otto-Preminger-Institut, était même allée jusqu'à accepter la saisie d'un film offensant pour la foi chrétienne, alors même que l'association qui en assurait la diffusion arguait du fait qu'elle avait prévu de projeter le film dans son cinéma, qui n'était accessible au public qu'après acquittement d'un droit d'entrée et qu'en outre, son public se composait, dans l'ensemble, de personnes intéressées par la culture progressiste. Elle avait par ailleurs, soutenait-elle, appliqué scrupuleusement la législation en vigueur, qui refusait l'accès du film aux mineurs de dix-sept ans. À quoi la Cour répondit que «bien que l'accès au cinéma pour voir le film litigieux fût soumis au paiement d'un droit d'entrée et à une condition d'âge, le film avait fait l'objet d'une large publicité. Le public avait une connaissance suffisante de son thème et de ses grandes lignes pour avoir une idée claire de sa nature; pour ces motifs, la projection envisagée doit passer pour avoir constitué une expression suffisamment "publique" pour être offensante» (§ 54). Quoiqu'il en soit, encore convenait-il de porter au préalable un jugement sur la réalité et la gravité de l'atteinte dont se plaignaient les croyants. D'une affaire à l'autre les avis des magistrats pouvaient diverger $^{12}$; du moins la Cour de cassation veillait-elle à leur objectivité par

11. V. en ce sens: TGI Paris, réf., 23 oct. 1984, Ave Maria: D. 1985, Jur., p. 31, note R. Lindon; Gaz. Pal. 1984, 2, p. 727, confirmé par CA Paris, 26 oct. 1984: Gaz. Pal. 1985, 1, p. 342, note Ph. Bertin.

12. V. TGI Paris, 20 janv. 1997: Les Petites Affiches, 24 févr. 1997, p. 10, note F. Gras: affaire du film Larry Flint, où l'affiche représente un homme debout les bras en croix et devant lui une femme en maillot de bain: pas d'interdiction dès lors qu'il ne s'agit que d'une allusion assez lointaine au Christ crucifié; TGI Paris, 21 févr. 2002: JCP G 2003, II 10064, note Ph. Malaurie: affaire du film Amen de Costa-Gavras, où l'affiche mêle croix gammée et croix chrétienne: l'affiche n'a pas été interdite faute de preuve de «la manifeste illicéité du trouble invoqué». 
un contrôle relativement sourcilleux de la motivation de leurs sentences ${ }^{13}$. C'est très exactement dans la mouvance de cette jurisprudence que s'inscrivirent, en 2005, le tribunal de grande instance et la cour d'appel de Paris ${ }^{14}$ dans une affaire où des affiches publicitaires parodiaient une fresque célèbre de Léonard de Vinci sur la dernière Cène, en substituant à Jésus et à ses apôtres un homme dos nu enlacé par des femmes aux attitudes lascives. Ayant l'une et l'autre estimé cette parodie gravement offensante pour les chrétiens, les deux juridictions fustigèrent tour à tour «le choix délibéré d'installer, dans un lieu de passage obligé pour le public, une affiche aux dimensions imposantes, qu'aucun regard ne peut éviter», ce qui constituait à leurs yeux «un acte d'intrusion agressive et gratuite dans le tréfonds des croyances intimes de ceux qui, circulant librement sur la voie publique et ne recherchant aucun contact singulier avec une ouvre ou un spectacle déterminé, se voient, hors de toute manifestation de volonté de leur part, nécessairement et brutalement confrontés à une manifestation publicitaire et commerciale ${ }^{15} »$. Elles autorisèrent en conséquence, à la demande de l'association "Croyances et libertés» représentée par le président de la Conférence des évêques de France, le retrait desdites affiches de l'espace public.

Las! C'était ne pas tenir suffisamment compte d'une jurisprudence amorcée presque cinq ans auparavant par l'Assemblée plénière de la Cour de cassation. Soucieuse de mieux protéger la liberté d'expression, la haute juridiction, dans sa formation la plus solennelle, y posait en principe que «les abus de la liberté d'expression prévus et réprimés par la loi du 29 juillet 1881 ne peuvent être réparés sur le fondement de l'article $1382 \mathrm{du}$ Code civil ${ }^{16} »$. Dès lors, lorsque le fait invoqué est une diffamation ou une injure, seuls les éléments constitutifs de ces infractions, tels que retenus par les articles 32 et 33 de la loi de 1881, autorisent, non seulement une condamnation pénale, mais encore une réparation civile ${ }^{17}$. De même, le juge des référés, saisi de demandes fondées

13. V. Cass. $1^{\text {re }}$ civ., 29 oct. 1990 : Bull. civ. I, no 226, La dernière tentation du Christ.

14. TGI Paris, 10 mars 2005 et CA Paris, 8 avr. 2005: D. 2005, p. 1326, note P. Rolland; JCP G 2005, II 10109, note Ph. Malaurie.

15. C'est la motivation du tribunal. Celle, plus sommaire, de la cour d'appel est dans le même sens.

16. Cass. ass. plén., 12 juill. 2000: BICC $1^{\text {er }}$ nov. 2000, concl. L. Joinet, rapp. F. DuRIEux; RTD civ. 2000, p. 845, obs. P. Jourdain.

17. V. Cass. $2^{\text {e }}$ civ., 29 mars 2001: Bull. civ. II, no 67 (fait diffamatoire); 20 nov. 2003: Bull. civ. II, $\mathrm{n}^{\circ} 347$ (faits injurieux et diffamatoires). V. aussi, à propos de la provocation à la haine religieuse: Cass. $2^{e}$ civ., 8 mars 2001: Bull. civ. II, $\mathrm{n}^{\circ} 47$, qui considère que seul constitue une faute civile le fait de provocation qui entre dans la définition de l'article 26, al. 5, de la loi de 1881 . 
sur une diffamation ou une injure, ne peut légitimement prononcer de mesures restrictives de la liberté d'expression que si le trouble invoqué devant lui est un fait injurieux ou diffamatoire au sens de la loi de $1881^{18}$. S'arc-boutant sur ces principes nouveaux, la première chambre civile de la Cour de cassation décida de casser l'arrêt rendu par la cour d'appel de Paris dans l'affaire de la parodie de la dernière Cène de Jésus. «La seule parodie de la forme donnée à la représentation de la Cène, qui n'avait pas pour objectif d'outrager les fidèles de confession catholique, ni de les atteindre dans leur considération en raison de leur obédience, ne constitue pas l'injure, attaque personnelle et directe dirigée contre un groupe de personnes en raison de leur appartenance religieuse », énonça-t-elle au visa cumulé des articles 29, alinéa 2 et 33, alinéa 3, de la loi du 29 juillet 1881, de l'article 809 du Code de procédure civile et 10 de la Convention européenne des droits de l'homme ${ }^{19}$. Ainsi, aux yeux de la Cour de cassation, l'injure pour motifs religieux, au sens de la loi du 29 juillet 1981, ne peut consister qu'en des «expressions outrageantes, des termes de mépris ou des invectives » adressées directement «à une personne ou un groupe de personnes en raison de leur appartenance à une religion déterminée». Dans le droit fil de la jurisprudence de sa chambre criminelle qui, à propos d'une affaire comparable, déclarait peu de temps auparavant que «les restrictions à la liberté d'expression sont d'interprétation étroite ${ }^{20}$ », la première chambre civile juge que les termes de la loi ne permettent pas d'appréhender les seules agressions verbales ou picturales envers la religion chrétienne. Un tel distinguo paraît pourtant assez byzantin. Où gît, en effet, la différence entre mépriser un catholique en raison de sa religion et moquer les croyances auxquelles adhère ce même catholique ${ }^{21}$ ? Il est difficile de le dire. Implicitement, la haute juridiction fait fond sur l'idée, énoncée ou approuvée par la chambre criminelle, selon laquelle les propos ou images

18. V. Cass. $2^{\mathrm{e}}$ civ., 7 mai 2002: Bull. civ. II, $\mathrm{n}^{\circ}$ 91; 22 janv. 2004: Bull. civ. II, $\mathrm{n}^{\circ} 21$.

19. Cass. $1^{\text {re }}$ civ., 14 nov. 2006: D. 2007, p. 2072, note E. Dreyer; JCP G 2007, II 10041, note Ph. Malaurie; Gaz. Pal. 2006, p. 3925, note P. Sarcelet.

20. Cass. crim., 14 mai 2006: Bull. crim., $\mathrm{n}^{\circ} 42$; Dr. pén. 2006, $\mathrm{n}^{\circ}$ 67, obs. M. VÉRON. V. aussi Cass. crim., 2 mai 2007: Bull. crim., $\mathrm{n}^{\circ} 115$.

21. Ainsi, pour reprendre des exemples prétoriens qui ne concernent pas les chrétiens mais tantôt les musulmans, tantôt les juifs, quelle différence peut-on faire entre ces deux formules: 1) «L'Islam est la religion la plus con»; 2) «Les juifs, c'est une secte, une escroquerie». Pourtant, la première n'a pas été considérée comme une injure, au sens pénal du terme (TGI Paris, ch. corr., 22 oct. 2002: Gaz. Pal. 2003, p. 3069, note P. BATTISTINI), alors que la seconde l'a été (Cass. crim., 15 mars 2005: Bull. crim., no 90 ; Dr. pén. 2005, no 85, obs. M. Véron; Gaz. Pal. 2005, 1, p. 1898, note Y. Monnet; Cass. ass. plén., 16 févr. 2007: JCP G 2007, II 10047, note E. Derieux; Dr. pén. 2007, no 50, note M. VÉRON). Mais il est vrai qu'elle fut prononcée par l'humoriste Dieudonné dont la réputation sulfureuse a pu incliner ses juges à se montrer moins cléments. 
injurieux pour la foi ne heurtent la sensibilité que de "certains chrétiens » ou «certains catholiques», entendant sans doute par-là les plus pieux d'entre eux. Mais au nom de quoi la haute juridiction s'érige-t-elle en juge de la foi des uns ou des autres? La Cour de cassation aurait voulu tarir les sources de la protection de la liberté religieuse dans notre pays qu'elle ne s'y serait pas prise autrement. Voici cette liberté purement et simplement expulsée du débat judiciaire. Malgré la grande marge d'appréciation qu'elle laisse aux États pour déterminer les restrictions à la liberté d'expression lorsqu'elle s'attaque aux convictions religieuses d'autrui ${ }^{22}$, il n'est pas sûr que la Cour de Strasbourg approuverait, si elle en était saisie, pareille mise au ban.

\section{LIBERTÉ RELIGIEUSE ET PRINCIPE DE LAÏCITÉ}

La France, on le sait, se veut un État laïque. Cette laïcité est fondamentalement conçue comme une neutralité vis-à-vis des choses de la religion. L'article $1^{\text {er }}$ de la Constitution de 1958, après avoir affirmé «La France est une République [...] laïque», énonce clairement qu'elle "assure l'égalité devant la loi de tous les citoyens sans distinction [...] de religion» et "respecte toutes les croyances». Même si cette scission a quelque chose d'artificiel et relève à ce titre davantage d'une fiction fondatrice ${ }^{23}$, l'on peut comprendre que l'État ne regarde qu'au bien commun temporel et prenne ses décisions indépendamment de tout critère défendu par une religion, quelle qu'elle soit. Mais le principe de laiicité est parfois érigé en modèle culturel, où tout ce qui regarde la chose publique exclut par définition une quelconque présence de la religion. L'on passe ainsi subrepticement de la neutralité religieuse à la neutralisation de la religion. Il y a là un refus implicite de tenir compte de l'histoire et des traditions du pays. La Cour européenne des droits de l'homme s'en arrange malgré tout assez bien. La prise en considération des traditions en question relève en effet à ses yeux de la marge d'appréciation des États ${ }^{24}$, libres par conséquent d'en tenir compte comme de les passer sous silence. Cette volonté typiquement française d'éradiquer de la chose publique tout relent de religiosité n'est pas nouvelle. Elle a par exemple inspiré certaines dispositions de la loi de 9 décembre 1905, tel l'article 28,

22. V. CEDH, 10 juill. 2003, n 44179/98, Murphy c. Irlande, § 67.

23. V. sur les rapports entre fiction et idéologie: M. DoAT, «Fictions juridiques et idéologies», in A.-B. CaIre (dir.), Les fictions en droit, Paris, LGDJ, 2015, p. 143 et s.

24. V. CEDH, Gde. ch., 18 mars 2011, no 30814/06, Lautsi c. Italie: RTD civ. 2011, p. 303, obs. J.-P. MARGUÉnAud (crucifix dans les classes des écoles publiques). 
selon lequel «il est interdit, à l'avenir, d'élever ou d'apposer aucun signe ou emblème religieux sur les monuments publics ou en quelque emplacement public que ce soit, à l'exception des édifices servant au culte, des terrains de sépulture dans les cimetières, des monuments funéraires, ainsi que des musées ou expositions». Mais, depuis quelques années, elle a repris de la vigueur dans deux domaines étroitement conjoints: les services publics et les lieux publics. Contrairement au passé, cette politique ne s'inspire plus principalement du souci de bouter la foi chrétienne hors de la sphère publique. Ce sont plutôt la foi et les mœurs musulmanes qui sont désormais visées. Il est clair cependant que ses effets sont susceptibles d'affecter toutes les religions, y compris celle fondée par le Christ.

Il y a beau temps que leur participation à la réalisation d'une activité de service public oblige tous ceux qui exercent de telles fonctions à mettre en berne leurs convictions religieuses pendant la durée de leur activité. Dans son arrêt Demoiselle Jamet du 3 mai 1950, le Conseil d'État évoquait déjà à ce propos «le devoir de stricte neutralité qui s'impose à tout agent collaborant au service public ${ }^{25} »$. La règle a été spécialement rappelée à l'adresse des agents du service de l'enseignement public par un avis Marteaux du Conseil d'État en date du 3 mai 200026. Une circulaire du 18 mai 2004 du ministre de l'Éducation nationale est venue préciser qu'elle interdisait «le port de tout signe d'appartenance religieuse, même discret» et imposait de «s'abstenir de toute attitude qui pourrait être interprétée comme une marque d'adhésion ou au contraire comme une critique à l'égard d'une croyance particulière ${ }^{27}$ ». Mais cette neutralité est requise de tout agent du service public, depuis les fonctionnaires en titre jusqu'à de simples salariés du secteur privé dans la mesure où ils collaborent à une mission de service public ${ }^{28}$. La Cour de Strasbourg admet sans difficulté cette position de la France. Elle en a même parfaitement exprimé les justifications. À propos du non-renouvellement du contrat de travail d'une assistante sociale dans un centre hospitalier public, en raison de son refus de s'abstenir de porter le voile musulman, la Cour souligne que «l'État qui emploie la requérante au sein d'un hôpital public peut juger nécessaire qu'elle ne fasse pas état de ses croyances religieuses dans l'exercice de ses fonctions pour garantir l'égalité de traitement des malades. [...] Une telle restriction trouve sa source dans le principe de laïcité de l'État, et celui

25. Lebon, p. 247.

26. RFDA 2001, p. 146, concl. R. SCHWARTZ.

27. JCP G 2004, III 20046.

28. V., par ex.: Cass. soc, 19 mars 2013: Dr. soc. 2013, p. 388, obs. E. Dockès: salariée intervenant dans le service "prestations maladie» de la Sécurité sociale. 
de neutralité des services publics, principes dont la Cour a déjà approuvé une stricte mise en œuvre lorsqu'il s'agit d'un principe fondateur de l'État ${ }^{29} »$. Certes, pourrait-on dire, la neutralité des services publics peut être garantie indépendamment du fait que les personnes appelées à y exercer leurs fonctions adhèrent ouvertement à telle ou telle confession religieuse; mais là n'est pas la question. Ce qui est en cause et qui justifie le strict devoir de réserve imposé à ces personnes, c'est « un principe fondateur» de l'État français. C'est, en définitive, une question d'image: le reflet d'un mythe. Voilà pourquoi même le simple port d'une tenue vestimentaire qui pointe en direction d'une religion doit être exclu, dès lors qu'il s'agit d'un service public en France. Peu importe, dans ces conditions, les fonctions remplies par l'intéressé(e) ${ }^{30}$. Ces considérations s'imposent-elles avec la même force lorsque l'on a affaire à de simples collaborateurs occasionnels du service public? La question se pose aujourd'hui avec acuité pour les parents qui acceptent d'accompagner les enfants d'une école publique lors des sorties scolaires. Estimant que les parents concernés participaient ainsi à une mission de service public, le tribunal administratif de Montreuil s'était prononcé en faveur de l'interdiction du port du voile islamique par des mères de famille accompagnatrices ${ }^{31}$. Mais le Conseil d'État, dans une étude demandée par le Défenseur des droits et publiée le 19 décembre 2013, estima pour sa part qu'en l'état du droit français, il était impossible d'identifier un statut de collaborateur du service public et que des restrictions à la liberté des parents accompagnateurs de manifester leurs opinions religieuses ne pouvaient résulter que de textes particuliers ou de considérations liées à l'ordre public ou au bon fonctionnement du service ${ }^{32}$. Le tribunal administratif de Nice s'est, depuis, rallié à cette opinion ${ }^{33}$. Le tollé provoqué par le vote récent, au Sénat, d'une proposition de loi visant à interdire le port de signes religieux aux accompagnateurs de sorties scolaires, montre en tout cas que la question n'est pas mûre ${ }^{34}$.

29. V. CEDH, 26 nov. 2015, no 64846/11, Ebrahimian c. France.

30. V., pour l'enseignement public: CE, avis, 3 mai 2000, Marteaux, précit.: «Il n'y a pas lieu d'établir une distinction entre les agents de ce service public selon qu'ils sont ou non chargés de fonctions d'enseignement. » Pour le service "prestations maladie» de la Sécurité sociale: Cass. soc., 19 mars 2013, précit.: «Peu importe que la salariée soit ou non directement en contact avec le public. » Certains arrêts sont cependant plus nuancés: CAA Lyon, 27 nov. 2003: AJDA 2004, p. 154, note F. Melleray: «Il y a lieu de tenir compte [...] de la nature des fonctions confiées à l'agent, ainsi que l'exercice, par lui, soit de prérogatives de puissance publique, soit de fonctions de représentation. »

31. TA Montreuil, 22 nov. 2011, $\mathrm{n}^{\circ}$ 1012015, Mme Osman.

32. Étude demandée par le Défenseur des droits le 20 septembre 2013 et adoptée par l'Assemblée générale du Conseil d'État le 19 décembre 2013, p. 30 et s.

33. TA Nice, 9 juin 2015, $\mathrm{n}^{\circ} 1305386$.

34. V. Le Monde, 29 oct. 2019. 
Quoiqu'il en soit, un point demeure certain: tout propos, toute attitude, tout signe qui manifeste extérieurement une appartenance religieuse est ici par principe (et non en principe) exclu. À l'heure où s'accroît régulièrement en France le nombre des dispositions légales qui renient ouvertement les préceptes moraux et religieux les plus fondamentaux (respect de la vie humaine du moment de la conception jusqu'à la mort, respect des lois de la sexuation et de la génération...), cette position totalisante et même à certains égards totalitaire place de plus en plus souvent les croyants devant la désolante alternative entre se soumettre et agir contre leur conscience, ou se démettre et perdre parfois un emploi dans lequel ils excellaient. Certes, dans la majorité des cas, cette situation peut être évitée avec un peu de prudence et d'entregent. Le juge qui ne veut pas appliquer une loi que ses convictions réprouvent, le médecin anesthésiste qui ne souhaite pas endormir une patiente pour que soit procédé à son interruption médicale de grossesse, peuvent toujours se déporter, quitte à obtenir de changer de juridiction ou de service. Mais ce ne sont là que des accommodements de fait. Tout accommodement de droit est-il donc exclu? Pas nécessairement. Dans certains cas particuliers, la loi a pu instaurer de véritables clauses de conscience, telle celle dont profitent encore aujourd'hui les médecins, sages-femmes, infirmiers ou infirmières lorsqu'ils doivent pratiquer une interruption de grossesse ${ }^{35}$. Mais, en dehors de ces cas, ne peut-on arguer de la liberté religieuse constitutionnellement et conventionnellement garantie, pour autoriser tel ou tel agent du service public à s'affranchir ponctuellement de son devoir de stricte neutralité? Telle est la question qui fut posée naguère au Conseil constitutionnel dans une affaire où, après l'entrée en vigueur de la loi du 17 mai 2013 autorisant le mariage entre personnes de même sexe, des maires soutenaient l'inconstitutionnalité des dispositions légales en ce qu'elles avaient omis de prévoir à leur profit une clause de conscience leur permettant de s'abstenir de célébrer ce genre de mariage. Pour justifier le rejet de la demande, les juges constitutionnels avancèrent d'abord «qu'en ne permettant pas aux officiers de l'état civil de se prévaloir de leur désaccord avec les dispositions de la loi du 17 mai 2013 pour se soustraire à l'accomplissement des attributions qui leur sont confiées par la loi pour la célébration du mariage, le législateur a entendu assurer l'application de la loi relative au mariage et garantir ainsi

35. V. CSP, art. L. 2212-8. L'on sait par ailleurs que la tentative du Conseil de l'ordre des pharmaciens d'introduire, au profit de ces personnels de santé, une clause de conscience destinée à "refuser d'effectuer un acte pharmaceutique susceptible d'attenter à la vie humaine», s'est soldée par un échec en raison du tollé médiatique qu'elle avait soulevé (V. Le Monde, 19 juill. 2016). 
le bon fonctionnement et la neutralité du service public de l'état civil. » Aux yeux du Conseil, tout dépend donc de la volonté du législateur, qui n'est jamais tenu d'accorder une clause de conscience à des agents du service public. Pour faire bonne mesure, les conseillers précisèrent ensuite «qu'eu égard aux fonctions de l'officier de l'état civil dans la célébration du mariage, [le législateur] n'a pas porté atteinte à la liberté de conscience ${ }^{36} »$. Qu'estce à dire, semble-t-il, sinon que maires et adjoints au maire doivent, dans l'exercice de leurs fonctions, mettre leurs consciences entre parenthèses? La seule liberté qui leur reste est alors justement celle de renoncer à exercer cette liberté. À eux de décider, en conscience, s'ils sont prêts, ou non, à agir contre leur conscience.

Le processus d'expulsion de toute référence religieuse concerne encore les « lieux publics». Telle qu'elle est employée en notre matière, cette notion est empreinte d'ambiguité. À la lettre, sont publics tous ces lieux où chacun est libre d'accéder, le cas échéant moyennant paiement. Mais il s'agit aussi, par extension, des lieux où, matériellement, se déroule une activité à caractère public. L'article 2 de la loi du 11 octobre 2010 interdisant la dissimulation du visage dans l'espace public dispose en ce sens que «l'espace public est constitué des voies publiques ainsi que des lieux ouverts au public ou affectés à un service public». Ainsi, à côté des rues, des places et des jardins publics, des salles publiques et des emplacements publics, il faut aussi compter sur les écoles publiques, les hôpitaux publics, les enceintes où se rend la justice et celles où les lois sont votées, les mairies, etc. Tous ces lieux doivent d'abord se caractériser par l'absence de tout symbole d'inspiration religieuse, comme l'a rappelé, dans le prolongement de l'article 28 de la loi du 9 décembre 1905, le Conseil d'État à propos de l'installation de crèches de Noël dans les mairies ${ }^{37}$. Mais le principe de laïcité conduit-il à imposer également à ceux qui ont accès à ces lieux publics, un devoir de réserve vestimentaire? Lorsqu'ils pénètrent dans un tribunal ou circulent dans la rue, un prêtre doit-il cesser de porter son clergyman ou sa soutane, un juif sa kippa, un sikh son turban et une musulmane son foulard? Voilà qui paraît pour le moins difficile à admettre. L'espace public ne devrait-il pas être le lieu où chacun est libre de se vêtir et de s'exprimer comme bon lui semble, sous la seule réserve de ne porter atteinte ni à l'ordre public, ni aux bonnes mours? De fait, c'est bien en ce sens que notre droit se prononce ordinairement ${ }^{38}$. Pourtant, lorsqu'il s'agit

36. Cons. const. 18 oct. 2013, no 2013-353 QPC, cons. 10.

37. 9 nov. 2016, no 395223.

38. V. A. SÉRIAuX, «L'espace public», in Droit et technique. Études Xavier Linant de Bellefonds, Paris, Litec, 2007, p. 433, no 5. 
du port de vêtements ou de signes religieux, il se montre plus rigoriste. S'il le fait parfois au nom d'une conception inédite de l'ordre moral, comme quand il dispose que «nul ne peut, dans l'espace public, porter une tenue destinée à dissimuler son visage »(art. $1^{\text {er }}$ de la loi du 11 octobre 2010) ${ }^{39}$, d'autres fois c'est en ayant recours au principe de laïcité. Ainsi, depuis la loi très controversée du 15 mars 2004 relative à l'application du principe de laïcité dans les écoles, collèges et lycées publics, l'article 141-5-1 du Code de l'éducation porte que «dans les écoles, les collèges et les lycées publics, le port de signes ou tenues par lesquels les élèves manifestent ostensiblement une appartenance religieuse est interdit ». L'interdiction n'a, il faut le reconnaître qu'une portée réduite. "La loi ne remet pas en cause le droit des élèves de porter des signes religieux discrets », indique en ce sens la circulaire d'application du 18 mai $2004^{40}$. Mais, contrairement à ce que l'on a parfois $\operatorname{dit}^{41}$, elle n'a pas qu'une valeur symbolique. Elle accroît sensiblement les pouvoirs de police du chef d'établissement et, si elle vise surtout les élèves de confession musulmane ${ }^{42}$, rien n'empêche de l'utiliser à l'adresse de jeunes chrétiens qui ne voudraient pas ou ne sauraient pas se tenir à la grande discrétion qu'on leur recommande. En revanche, la France n'est jamais allée jusqu'à étendre la même prohibition dans les enceintes, beaucoup plus libérales il est vrai, de l'université publique ${ }^{43}$. Et si l'instruction générale du bureau de l'Assemblée nationale comporte bien l'interdiction, à l'adresse des visiteurs, du port de signes religieux ostensibles, il n'en est pour l'instant rien s'agissant des

39. Dans sa décision du 7 octobre 2010 ( $\mathrm{n}^{\circ} 2010-613$ DC) pour admettre la conformité de cette loi à la Constitution, le Conseil constitutionnel ne se réfère pas au principe de laiicité, mais à une certaine conception de la vie en société, où le visage est le reflet de la personne. La Cour EDH (Gde. ch., $1^{\text {er }}$ juill. 2014, $n^{\circ}$ 43835/11, S.A.S. c. France) a retenu une analyse comparable à l'heure de rejeter le recours pour contrariété à la Convention. L'interdiction fulminée par la loi française est justifiée, dit-elle, par la préservation des conditions du «vivre ensemble».

40. Précit.

41. V. Ph. Malaurie, «Laïcité, voile islamique, réforme législative», JCP G 2004, I 124.

42. C'est à leur sujet que sont intervenues les premières discussions portées devant les juridictions administratives, autour de la participation aux activités obligatoires d'éducation physique et sportive. La Cour européenne des droits de l'homme a, depuis, validé les décisions d'interdiction du port du voile islamique et autres tenues féminines comme inadaptées aux cours d'éducation physique et sportive: CEDH, 4 déc. 2008, nº 27058/05, Dogru c. France et no 31645/04, Kervanci c. France: RTD civ. 2009, p. 285, obs. J.-P. MARGUÉNAUD. V. aussi, à propos du refus des parents musulmans que leurs filles participent aux cours obligatoires de natation: CEDH, 10 janv. 2017, n 29086/12, Osmanoglu et Kocabas c. Suisse.

43. Contrairement par exemple à la Turquie. V. CEDH, 29 juin 2004, no 44774/98, Leyla Şahin c. Turquie: D. 2005, p. 204, note G. YILDIRIM (port du foulard islamique par une étudiante en médecine dans l'enceinte universitaire). 
salles d'audience de nos juridictions ${ }^{44}$. Il reste que la Cour de Strasbourg, qui a déjà connu de ce genre de prohibition dans d'autres États, n'exclut pas de les valider dès lors qu'elles s'inspirent du souci de faire primer, dans un établissement public, le respect de la neutralité sur le libre exercice du droit de manifester sa religion ${ }^{45}$. Elle s'était d'ailleurs déjà prononcée dans le même sens s'agissant de la conventionnalité des dispositions de la loi du 15 mars $2004^{46}$. Affaire à suivre, donc, mais qui n'augure encore rien de bon pour la liberté religieuse dans notre pays.

44. Rappr., sur la question du port de signes religieux par les avocats dans l'exercice de leur activité judiciaire: B. BONNET, J. FERRON, «Le port de signes religieux par les avocats», JCP G 2018, doctr. 781.

45. V. CEDH, 18 sept. 2018, no 3413/09, Lachiri c. Belgique, dans lequel la Cour souligne qu' ' un tribunal est [...] un établissement "public" dans lequel le respect de la neutralité à l'égard des croyances peut primer sur le libre exercice du droit de manifester sa religion, à l'instar des établissements d'enseignement publics» (§ 45). Il est vrai qu'il ne s'agit là que d'une réponse à un argument qui soutenait qu'un tribunal est un lieu public similaire à une voie ou une place publique. Il s'agissait dans cette affaire de l'une des parties au procès, qui s'était vue interdire sa présence à l'audience parce qu'elle portait le foulard islamique. Comp. avec CEDH, 5 déc. 2017, nº 57792/15, Hamidović c. BosnieHerzégovine, où il s'agissait d'un témoin, condamné pour outrage à magistrat pour avoir refusé d'enlever sa calotte lors de sa comparution à l'audience.

46. V. CEDH, 17 juill. 2009, no 43563/08, Aktas c. France; no 14308/08, Bayrak c. France; $n^{\circ}$ 18527/08, Gamaleddyn c. France; n ${ }^{\circ}$ 29134/08, Ghazal c. France; no 25463/08, J. Singh c. France et $\mathrm{n}^{\circ} 27561 / 08$, R. Singh c. France. V. aussi, pour la Turquie: CEDH, 24 janv. 2006, $\mathrm{n}^{\circ}$ 26625/02, Köse et autres c. Turquie. 